\title{
CARACTERE ELLIPTIQUE ET OPÉRATIONS COHOMOLOGIQUES *
}

\author{
ABDELAZIZ KHELDOUNI - FAYÇAL LAMRINI \\ Université Mohammed Benabdallah, Maroc
}

\begin{abstract}
Theorem 4.6 of $[6]$ gives the expression of Todd character in terms of Landweber-Novikov cohomology operations. In this paper we generalise this result to any I-oriented cohomology theory, and express elliptic character by means of Landweber-Novikov operations.
\end{abstract}

*Recherche menée dans le cadre du Programme d'Appuiá la Recherche Scientifique (PARS) MI33. 


\section{Introduction}

L.Smith a établi une relation entre le caractère de Todd

$$
t h: M U_{*}(X) \rightarrow H_{*}\left(X ; \pi_{*}(K)\right)
$$

et les opérations de Landweber-Novikov :

$$
\theta(\alpha)=\sum_{E} \mu\left(\left(\chi s_{E}\right)(\alpha)\right) \tilde{B}_{E}
$$

(cf. [6] Theorem 4.6) pour tout $\alpha \in M U_{*}(X)$, où $s_{E}$ est l'opération de Landweber-Novikov relative au multi-indice á support fini $\mathrm{E}$, et $\chi$ est l'anti-involution de l'algèbroïde de Hopf $M U^{*}(M U)$. Notons que le formalisme utilisé dans [6] pour exprimer le caractère de Todd en fonction des opérations de Landweber-Novikov reste valable pour une théorie cohomologique $I$-orientée à la place de la K-théorie. On obtient alors une expression du caractère d'un genre multiplicatif $\varphi$, de série caractéristique $\sum_{k \geq 0} a_{k}^{\varphi} X^{k}$, en fonction des opérations de Landweber- Novikov, qui généralise le Théorème 4.6 de [6] à une théorie cohomologique $I$-orientée quelconque :

Théorème 3.4 : Soient $\alpha \in M U_{*}(X), E=\left(e_{1}, e_{2}, \ldots\right)$. En notant $a_{E}^{\varphi}=\left(a_{1}^{\varphi}\right)^{e_{1}}\left(a_{2}^{\varphi}\right)^{e_{2}} \ldots$ nous avons:

$$
\mathcal{C}^{\varphi}(\alpha)=\sum_{E} \mu\left(\left(\chi s_{E}\right)(\alpha)\right) a_{E}^{\varphi}
$$

L'application de ce résultat à la cohomologie elliptique permet alors d'avoir une expression du caractère elliptique en fonction des opérations de Landweber-Novikov :

Proposition 4.2 : Pour tout $\alpha \in M U_{*}(X)$, nous avons :

$$
e(\alpha)=\sum_{E} \mu\left(\left(\chi s_{E}\right)(\alpha)\right) \tilde{H}_{E}
$$

où les $\tilde{H}_{E}$ sont définis à partir de la série caractéristique du genre elliptique.

$\mathrm{Ce}$ dernier résultat appelle quelques commentaires. En effet, dans notre version de la cohomologie elliptique, le groupe des coefficients est $\mathbb{Z}\left[\frac{1}{2}\right]\left[\delta, \epsilon, \gamma^{-1}\right]$ où $\gamma=\left(\delta^{2}-\epsilon\right)$, qui est un anneau $4 X$-gradué. De plus, si on pose $A *=\mathscr{Z}\left[\frac{1}{2}\right]\left[\delta, \epsilon, \gamma^{-1}\right]$, la proposition 1.2 de [3] donne $A_{8 k}=\gamma^{k} \mathbb{Z}\left[\frac{1}{2}\right]\left[\delta^{2} / \gamma\right]$ et $A_{8 k+4}=\delta A_{8 k}$; il serait alors intéressant d'écrire la formule de la proposition 3.4 comme une expression polynomiale en $\delta, \epsilon$ à coefficients dans $\boldsymbol{Z}\left[\frac{1}{2}\right]\left[\delta^{2} / \gamma\right]$. Ceci peut se faire au prix de l'introduction de polynômes assez compliqués, dont l'étude et l'interprétation restent à faire. 


\section{Remarques sur le genre multiplicatif}

Soit $F(X, Y)$ un groupe formel sur une $I$-algèbre graduée $A_{*} . \mathrm{F}$ est classifié par un homomorphisme d'anneaux $\varphi: M U_{*} \rightarrow A_{*}$ (genre multiplicatif) tel que $F(X, Y)=\varphi_{*} F^{U}(X, Y)$, où $F^{U}$ est le groupe formel universel et $M U$ est le spectre de Milnor. Si $g_{F}$ est le logarithme de $F(X, Y)$ la série formelle $Q(X)=\frac{X}{g_{F}^{-1}(X)}$ définit une m-suite de Hirzcbruch $K^{\varphi}=\left\{K_{i}^{\varphi}\left(c_{1}, \ldots, c_{i}\right)\right\} \in H^{* *}\left(B U ; A^{*}\right)$ (cf.[2]). Nous avons alors pour toute classe de bordisme $\left\{M T \in M U^{*}\right.$ :

$$
\varphi(\{M\})=\left\langle\sum_{i \geq 0} K_{i}^{\varphi}\left(c_{1}(\tau M), \ldots, c_{i}(\tau M)\right), \sigma_{M}^{I I}\right\rangle
$$

où $\tau M$ est le fibré tangent stable de $M$ et $\sigma_{M}^{H}$ la classe fondamentale de $M$ en homologie ordinaire. Rappelons aussi que si $j: \mathbb{C} \mathrm{P}^{n} \rightarrow \mathbb{C} \mathrm{P}^{\infty}=$ $B U(1) \rightarrow B U$ est l'inclusion naturelle, et si $\beta_{n}=j_{*}\left(\sigma_{\mathbb{C}}^{H} \mathrm{P}^{n}\right) \in H_{2 n}(B U ;) h$ alors $H_{*}\left(B U: \not{A}\left[\beta_{1}, \beta_{2}, \ldots\right]\right.$, ct les monônes $\beta^{E}=\beta_{1}^{e_{1}} \beta_{2}^{e_{2}} \ldots \beta_{r}^{e_{r}}$ avec $E=$ $\left(e_{1}, \ldots, e_{r}\right), e_{i} \geq 0$ forment une $I$-base de $H_{*}\left(B U ; L\right.$. Si en plus $\Phi_{*}$ : $H_{*}(M U ;) I \rightarrow H_{*}(B U ;) I$ cst l'isomorphisme de Thom, en prenant $b_{i}=\Phi_{*}^{-1}\left(\beta_{i}\right)$ , nous avons : $H_{*}\left(M U ; \mid I \simeq \mathbb{Z} b_{1}, b_{2}, \ldots\right]$. La classe exponentielle $K^{\varphi}(c)=$ $1+K_{1}^{\varphi}\left(c_{1}\right)+K_{2}^{\varphi}\left(c_{1}, c_{2}\right)+\ldots \in H_{* *}\left(B U ; A_{*}\right)$ peut-ôtre identifiée à un ćlément de $H o n\left(H_{*}(M U ; \mathbb{X}) ; A_{*}\right)$ comme suit :

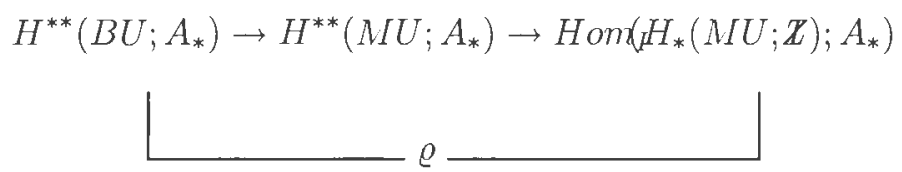

où $\varrho\left(K^{\varphi}\right): H_{*}\left(M U ; I \rightarrow A_{*}\right.$ est le $I$-homomorphisme qui à $x$ associc $\varrho\left(K^{\varphi}\right)(x)=\left\langle\Phi^{*}(c) ; K^{\varphi}(c) x\right\rangle$

Proposition 2.1 : Le diagramme suivant est commutatif:

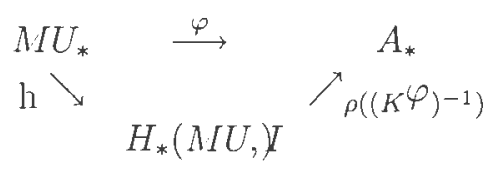

où $h: \pi_{*}(M U) \rightarrow \pi_{*}(\mathcal{H} A \wedge M U)$ est l'homomorphisme d'Hurewicz; et $\mathcal{H} A$ le spectre de Mac-Lane pour l'anneau gradué $A_{*}$.

Démonstration : Précisons d'abord quelques notations. Si $M^{2 n}$ est une U-varieté de dimension $2 \mathrm{n}$, la classe $\left\{M^{2 n}\right\} \in M U_{2 n}=\pi_{2 n}(M U)=$ 
$\left[S^{2 n+2 k} ; M U(k)\right]$ (k grand) est représentée par $\tilde{f}$ op où $\tilde{f}: M_{\nu} \rightarrow M U(k)$ est l'application induite sur les espaces de Thom par l'application $f: M^{2 n} \rightarrow$ $B U(k)$ classifiant le fibré normal $\nu$ pour le plongement $M^{2 n} \rightarrow S^{2 n+2 k}$, et où $p: S^{2 n+2 k} \rightarrow M_{\nu}$ est l'application obtenue à l'aide de la construction de Thom (cf.[7]). Cependant, $h\left(\left\{M^{2 n}\right\}\right)=\tilde{f} o p_{*}\left(\iota_{2 n+2 k}\right), \iota_{2 n+2 k}$ ćtant la classe fondamentale de $S^{2 n+2 k}$. Nous avons aussi le diagramme commutatif:

$$
\begin{array}{cccc}
H_{2 n+2 k}\left(S^{2 n+2 k} ; A_{*}\right) \stackrel{p_{*}}{\rightarrow} H_{2 n+2 k}\left(M_{\nu} ; A_{*}\right) & \stackrel{\tilde{f}_{*}}{\rightarrow} & H_{2 n+2 k}\left(M U(k) ; A_{*}\right) \\
& \Phi_{*} \downarrow & & \downarrow \Phi_{*} \\
& H_{2 n}\left(M^{2 n} ; A_{*}\right) & \stackrel{f_{*}}{\rightarrow} & H_{2 n}\left(B U(k) ; A_{*}\right)
\end{array}
$$

Notons que $\Phi_{*} o p_{*}$ envoie le générateur canonique $\iota_{2 n+2 k} \in$ $H_{2 n+2 k}\left(S^{2 n+2 k} ; A_{*}\right)$ sur la classe fondamentale $\sigma_{M I}^{H}$ de $M^{2 n}$. Si on note $\gamma$ le fibré universel sur $B U$, nous avons :

$$
\begin{aligned}
\rho\left(\left(K^{\varphi}\right)^{-1}\right)\left(h\left(\left\{M^{2 n}\right\}\right)\right) & =\left\langle\Phi^{*}\left(\left(K^{\varphi}(\gamma)\right)^{-1}\right), \tilde{f}_{*} o p_{*}\left(\iota_{2 n+2 k}\right)\right\rangle \\
& =\left\langle\left(K^{\varphi}(\gamma)\right)^{-1}, \Phi_{*} o \tilde{f}_{*} o p_{*}\left(\iota_{2 n+2 k}\right)\right\rangle \\
& =\left\langle\left(K^{\varphi}(\gamma)\right)^{-1}, f_{*} O \Phi_{*} o p_{*}\left(\iota_{2 n+2 k}\right)\right\rangle \\
& =\left\langle\left(K^{\varphi}(\gamma)\right)^{-1}, f_{*}\left(\sigma_{M}^{H}\right)\right\rangle=\left\langle f^{*}\left(K^{\varphi}(\gamma)\right)^{-1}, \sigma_{M}^{I I}\right\rangle \\
& \left.\left.=\left\langle\left(K^{\varphi}(\nu M)\right)^{-1}, \sigma_{M I}^{I I}\right)\right\rangle=\left\langle\left(K^{\varphi}(\tau M)\right)^{-1}, \sigma_{M I}^{I I}\right\rangle\right\rangle \\
& =\varphi(M) .
\end{aligned}
$$

Corollaire 2.2 : La série formelle

$$
\mathcal{Q}^{\varphi}(X)=1+\sum_{i \geq 1} \rho\left(K^{\varphi}\right)\left(b_{\imath}\right) X^{\imath}
$$

est la série caractéristique du genre multiplicatif $\varphi$.

Démonstration : Rappelons que la m-suite de Hirzebruch $K^{\varphi}=\left\{K_{i}^{\varphi}\right\}$ d'un genre $\varphi$ et sa série caractéristique $\mathcal{Q}^{\varphi}(X)$ sont liées do la manière suivante: soit $\xi$ un fibré véctoriel complexe de rang $n$, en écrivant formellement sa classe totale de Chern sous la forme $c(\xi)(X)=\sum_{0 \leq i \leq n} c_{\imath}(\xi) X^{\imath}=$ $\prod_{1 \leq i \leq n}\left(1+t_{\imath} X^{\imath}\right)$ avec $c_{\imath}(\xi)=\sigma_{\imath}\left(t_{1}, \ldots, t_{n}\right) ;$ alors on a : $K^{\varphi}(c(\xi))=1+$ $\sum_{i \leq 1} K_{i}^{\varphi}\left(c_{1}(\xi), \ldots, c_{i}(\xi)\right) X^{i}=\prod_{1 \leq i \leq n} K^{\varphi}\left(1+t_{i} X\right)=\prod_{1 \leq i \leq n} \mathcal{Q}^{\varphi}\left(t_{i} X\right)$. Soit maintenant $\gamma_{1}$ le fibré de Hopf sur $\mathbb{C} \mathrm{P}^{\infty} \simeq B U(1)$ et $t=c_{1}\left(\gamma_{1}\right)$, on a : 
$\mathcal{Q}^{\varphi}(t X)=1+K_{1}^{\varphi}(t) X+K_{2}^{\varphi}(t, 0) X^{2}+\ldots$ avec $K_{i}^{\varphi}(t, 0 \ldots, 0) \in H^{2 i}\left(C \mathrm{P}^{\infty} ; \not t\right.$. $K_{i}^{\varphi}(t, 0, \ldots, 0)=\left\langle K_{i}^{\varphi}(t, 0, \ldots, 0) ; \sigma \mathbb{C P}_{\mathrm{P}^{i}}\right\rangle t^{2}$. Or si $\mathrm{j} \cdot \mathbb{C} \mathrm{P}^{\imath} \rightarrow \mathbb{C}^{\prime} \mathrm{P}^{\infty}$ est l'inclusion canonique, l'homomorphisme

$$
\begin{array}{clc}
H^{2 i}\left(\mathbb{C} \mathrm{P}^{\infty}, \boldsymbol{Z}\right) & \longrightarrow & I \\
x & \longrightarrow\langle x, \sigma & \left.\mathbb{C} \mathrm{P}^{i}\right\rangle
\end{array}
$$

est un isomorphisme. Ainsi, grace à cette identification, il suffit de montrer que $\rho\left(K^{\varphi} c(\gamma)\right)\left(b_{i}\right)=\left\langle K_{i}^{\varphi}\left(c_{1}, 0, \ldots, 0\right), \sigma \sigma \mathrm{CP}^{i}\right\rangle$ où $c_{1}$ est la première classe de Chern du fibré universel $\gamma$ sur BU. On a donc :

$$
\begin{aligned}
\rho\left(K^{\varphi} c(\gamma)\right)\left(b_{i}\right) & =\left\langle K^{\varphi} c(\gamma), \beta_{i}\right\rangle=\left\langle K_{i}^{\varphi}\left(c_{1}, \ldots, c_{i}\right), j_{*}\left(\mathbb{C P}^{i}\right)\right\rangle \\
& =\left\langle j^{*} K_{i}^{\varphi}\left(c_{1}, \ldots, c_{i}\right), \mathscr{C} \mathrm{P}^{i}\right\rangle=\left\langle K_{i}^{\varphi}\left(c_{1}\left(j^{*} \gamma^{1}\right), 0, \ldots, 0\right), \mathscr{C} \mathrm{P}^{i}\right\rangle
\end{aligned}
$$

La dérnière égalité provient du fait que l'inclusion $\mathrm{j}: \mathbb{C} \mathrm{P}^{i} \rightarrow B U(1) \rightarrow B U$ classifie le fibré de Hopf sur $\mathbb{C}^{\mathrm{P}}{ }^{i}$. Ainsi : $\rho\left(K^{\varphi}\left(b_{i}\right)\right)=\left\langle K_{i}^{\varphi}\left(c_{1}, 0, \ldots, 0\right), \mathbb{C}^{\top} \mathrm{P}^{i}\right\rangle$.

\section{Caractère d'un genre multiplicatif}

Nous supposons maintenant que $A_{*}$ est une $I$-algèbre graduée munie d'un groupc formel $F(X, Y)$ vérifiant le thćorème du foncteur exact de Landwebor [5]. L'application classifiante de $F(X, Y), \varphi: M U_{*} \rightarrow A_{*}$ est un genre multiplicatif qui confère à $A_{*}$ une structure de $M U_{*}$-module, et $h^{*}(X)=M U^{*}(X) \otimes_{\varphi} A_{*}$ est une théorie cohomologique multiplicative $I$-orientée dont l'anneau de base est $h_{*}(p t)=A_{*}$. Soit $E$ le spectre représentant la théorie $h^{*}(-)$ et $\nu: M U \rightarrow E$ son application classifiante. On sait que $\nu_{*}(p t)=\varphi$. Nous définissons le caractère du genre $\varphi$ (ou le $\varphi$-caractère) $C^{\varphi}$ par :

$$
C^{\varphi}=c h_{d} O \nu: M U \rightarrow \mathcal{H}\left(A_{)_{*}}\right.
$$

où $c h_{d}$ est le caractère de Chern-Dold (voir [1]).

Cette application de spectre-anneaux induit une transformation naturelle encore notée $C^{\varphi}$ :

$$
C^{\varphi}: M U_{*}(X) \rightarrow H_{*}(X ; A) *
$$

c'est à dire qu'en chaque degré n, nous avons:

$$
\begin{aligned}
C^{\varphi}: M U_{n}(X) & \longrightarrow \sum_{i=0}^{n} H_{n-i}\left(X, A_{i} \otimes Q\right) \\
\alpha & \longrightarrow C^{\varphi}(\alpha)=C_{n}^{\varphi}(\alpha)+C_{n-1}^{\varphi}(\alpha)+\ldots+C_{0}^{\varphi}(\alpha)
\end{aligned}
$$


où $C_{n-i}^{\varphi}(\alpha) \in H_{n-i}\left(X, A_{i}\right.$ est donné par l'équation suivante : $\alpha$ étant représenté par $\{M, f\}$, pour tout $x \in H^{n-i}\left(X ;, A_{i}\right.$

$$
\left\langle x, C^{\varphi}(\alpha)\right\rangle=\left\langle f^{*}(x) K^{\varphi}(c(\tau M)), \sigma_{M}^{I I}\right\rangle
$$

Notons que :

1) $C_{n}^{\varphi}(\alpha)=\mu(\alpha)$ où $\mu: M U_{*}(X) \rightarrow H_{*}\left(X ; A_{*}\right)$ est l'homomorphisme de Thom.

2) $C_{0}^{\varphi}(\alpha)=\varphi(\{M\})$

3) Si on prend dans (2) $X=p t$, on obtient :

$C^{\varphi}(p t): M U_{*} \longrightarrow A_{*}$

$\{M\} \longrightarrow \varphi(\{M\})$

Considérons maintenant l'homomorphisme

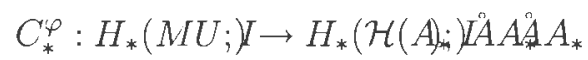

induit par (1) en homologie ordinaire à coefficients dans $I$.

Proposition 3.1 : L'homomorphisme $C_{*}^{\varphi}$ coïncide avec $\rho\left(K^{\varphi}\right) \otimes 1_{I}$

Démonstration : Nous avons besoin du lemme suivant :

Lemme 3.2 : Soient $E, F, H$ trois spectres et $G$ un spectre anneau. Si $f: E \rightarrow F$ et $g: G \rightarrow H$ sont deux applications de spectres, on peut considérer, d'une part les homomorphismes

$f_{*}^{G}: G_{*}(E) \rightarrow G_{*}(F):,: f_{*}^{G}: H_{*}(E) \rightarrow H_{*}(F):,: g_{*}: G_{*}(-) \rightarrow H_{*}(-)$, et d'autre part les homomorphismes d'Hurewicz $h^{E}:_{*}(E) \rightarrow G_{*}(E):,: h^{F}:{ }_{*}(F) \rightarrow G_{*}(F)$.

Nous avons alors le diagramme commutatif :

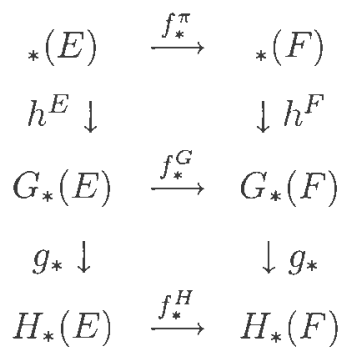

Il suffit alors de pendre dans le lemme $3.2 E=M U, F=\mathcal{H} A_{*}$ et $f=$ $C^{\varphi}: M U \rightarrow \mathcal{H} A_{*} ; G=\mathcal{H} X, H=\mathcal{H Q}$ et $g: \mathcal{H X} \rightarrow \mathcal{H} Q$. On aura donc $f_{*}^{H}=\rho\left(K^{\varphi}\right) \otimes 1_{I}$ 
Cependant, si $\sum_{k \leq 0} a_{E}^{\varphi} X^{k}$ est la série caractéristique du genre $\varphi$, on déduit à partir de la proposition 3.1 et du corollaire 2.2 l'expression de $\mathcal{C}_{*}^{\varphi}$ sur les générateurs $b_{k}$ de $H_{2 k}(M U ;) I$ Nous avons :

$$
\mathcal{C}_{*}^{\varphi}\left(b_{k}\right)=\rho\left(K^{\varphi}\right) \otimes 1\left(p_{k}\right)=a_{E}^{\varphi} \quad \in A Q
$$

D'autre part, la proposition 4.3 de [6] se généralise sans difficultés au cas d'une théorie cohomologique $I$-orientée. Notons d'abord que l'homomorphisme d'Hurewicz $h: M U_{*}(X) \rightarrow H_{*}(X ;) \otimes H_{*}(M U ;$; Induit par l'application de spectres $X^{+} \wedge M U \rightarrow X^{+} \wedge M U \wedge \mathcal{H}$ kst donné par (voir Proposition 4.3 de $[6])$ :

$$
h(\alpha)=\sum_{E} \mu\left(\left(\chi^{s_{E}}\right)(\alpha)\right) \otimes b_{E}
$$

où $s_{E}$ est l'opération de Landweber-Novikov relative à $E=\left(e_{1}, e_{2}, \ldots\right)$ et $\chi$ l'anti-involution de l'algèbre $M U^{*}(M U)$.

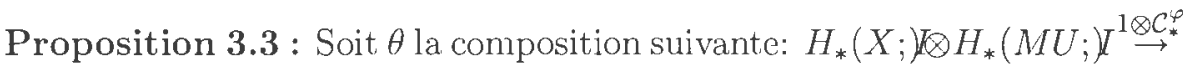
$H_{*}(X ;) \otimes H *\left(\mathcal{H} A_{*} ;\right) I \stackrel{I}{A} H_{*}\left(X ; I \otimes A_{*} \stackrel{\circ}{A} H_{*}\left(X ; A_{*}\right)\right.$. L'homomorphisme (2) est donné par: $\mathcal{C}_{*}^{\varphi}=\theta o h, h$ étant l'homomorphisme de Hurewicz (4).

Démonstration : Le diagramme :

$$
\begin{array}{cc}
X^{+} \wedge M U \wedge S^{0} \stackrel{1 \wedge 1 \wedge \iota \mathcal{H} I}{\longrightarrow} X^{+} \wedge M U \wedge \mathcal{H} I \\
1 \wedge \mathcal{C}^{\varphi} \wedge 1 \downarrow & \downarrow 1 \wedge \mathcal{C}^{\varphi} \wedge 1 \\
X^{+} \wedge \mathcal{H} A_{*} \wedge S^{0} \stackrel{1 \wedge 1 \wedge \iota \mathcal{H} I}{\longrightarrow} X^{+} \wedge \mathcal{H} A_{*} \wedge \mathcal{H} I
\end{array}
$$

est évidemnent commutatif. En appliquant le foncteur $\pi_{*}(-)$, on obtient le diagramme commutatif:

$$
\begin{array}{ccc}
M U_{*}(X) & \stackrel{h^{M U \wedge X}}{\longrightarrow} & H_{*}(X \wedge M U ; Y \\
*\left(1 \wedge \mathcal{C}^{\varphi}\right) \downarrow & & \downarrow\left(1 \wedge \mathcal{C}^{\varphi}\right)_{*} \\
H_{*}\left(X ; A_{*}\right) & \underset{h^{\mathcal{H A *}} \longrightarrow X}{\longrightarrow} & H_{*}\left(X \wedge \mathcal{H} A_{*} ; I\right.
\end{array}
$$

d'où le résultat. 
Théorème 3.4 : Soient $\alpha \in M U_{*}(X), E=\left(e_{1}, e_{2}, \ldots\right)$.

Notons $a_{E}^{\varphi}=\left(a_{1}^{\varphi}\right)^{e_{1}}\left(a_{2}^{\varphi}\right)^{e_{2}} \ldots$ nous avons:

$$
\mathcal{C}^{\varphi}(\alpha)=\sum_{E} \mu\left(\left(\chi s_{E}\right)(\alpha)\right) a_{E}^{\varphi}
$$

\section{Démonstration :}

$$
\begin{aligned}
\mathcal{C}^{\varphi}(\alpha) & =\theta \text { oh }(\alpha), \quad \text { d'après la proposition } 3.3 \\
& =\theta\left(\sum_{E} \mu\left(\left(\chi^{s_{E}}\right)(\alpha)\right) b_{E}^{\varphi}\right) \quad \text { d'aprés (4) } \\
& =\sum_{E} \mu\left(\left(\chi s_{E}\right)(\alpha)\right) \mathcal{C}_{*}^{\varphi}\left(b_{E}\right)=\sum_{E} \mu\left(\left(\chi^{s} s_{E}\right)(\alpha)\right) \prod_{k} \mathcal{C}_{*}^{\varphi}\left(b_{k}\right)^{e_{k}} \\
& =\sum_{E} \mu\left(\left(\chi s_{E}\right)(\alpha)\right) \prod_{k}\left(a_{k}^{\varphi}\right)^{e_{k}} \quad \text { d'aprés }(3) .
\end{aligned}
$$

\section{Applications}

\section{a) Caractère de Todd}

Si la théorie $h^{*}(-)$ est la K-théorie complexe, le $\varphi$-caractère est le celui de Todd [1], th: $M U_{*}(X) \rightarrow H_{*}\left(X ; \pi_{*}(K)\right)$. D'autre part, la théorie $K^{*}(-)$ est classifiée par le genre multiplicatif

$$
\varphi_{K}: M U_{*} \rightarrow A_{*}=\mathbb{Z}\left[u, u^{-1}\right]
$$

donné par $\varphi_{K}\left(\left\{M^{2 n}\right\}\right)=\operatorname{Td}\left(\left\{M^{2 n}\right\}\right) u^{n}$ où $T d\left(\left\{M^{2 n}\right\}\right)$ est le genre de Todd $T d: M U_{*} \rightarrow \mathcal{Z}$ de série caractéristique

$$
\mathcal{Q}_{T d}(X)=1+\frac{1}{2} X+\sum_{k \geq 1}(-1)^{k-1} \frac{B_{k}}{(2 k) !} X^{2 k}
$$

où les $B_{k}$ sont les nombres de Beroulli (voir [2]). Cependant, la série caractéristique de $\varphi_{K}$ est donnée par :

$$
\tilde{\mathcal{Q}}_{T d}(X)=1+\frac{1}{2} u X+\sum_{k \geq 1}(-1)^{k-1} \frac{B_{k}}{(2 k) !} u^{k} X^{2 k}
$$


Il découle alors du théorème 3.4 que pour tout $\alpha \in M U_{*}(X)$ :

$$
\operatorname{th}(\alpha)=\sum_{E} \mu\left(\left(\chi s_{E}\right)(\alpha)\right) \tilde{B}_{E}
$$

où $\tilde{B}_{E}=\left(\frac{u}{2}\right)^{e_{1}}\left(\bar{B}_{1} u^{2}\right)^{e_{2}} 0^{e_{3}}\left(\bar{B}_{2} u^{4}\right)^{e_{4}} \ldots 0^{e_{2 i+1}}\left(\bar{B}_{i+1} u^{2 i+2}\right)^{e_{2 i+2}} \ldots$ avec $E=\left(e_{1}, e_{2}, \ldots\right) \in \mathbb{N}^{\infty}$, et la convention $0^{0}=1$.

Remarque 4.1: Si la théorie $h^{*}(-)$ considérée est périodique, et si l'élément de póriodicité $u$ est de degré 2, alors à partir du groupe formel $F(X, Y)$ sur $A_{*}$, définissant $h^{*}(-)$, on pout considérer le groupe formel $F_{0}(X, Y)=$ $u F(X / u, Y / u)$ qui sera un groupe formel sur $A_{0}$. De plus son logarithme $g_{0}(X)$ est lié à celui de $F(X, Y)$ par $g(X)=(1 / u) g_{0}(u X)$. On déduit que si $\varphi: M U_{*} \rightarrow A_{*}$ est le genre associć au groupe formel gradué $F(X, Y)$, alors pour toute classe de bordisme $\{M\} \in M U_{2 k}$, on a $\varphi(\{M\})=\varphi_{0}(\{M\}) u^{k}$ , où $\varphi_{0}: M U_{*} \rightarrow A_{0}$ est l'application quiclassifiele groupe formel $F_{0}(X, Y)$. Dans ce cas nous avons une autre expression de $(3):$ Si $\left\{M^{2 k}\right\}$ est le générateur de $M U_{*}$ qui s'envoie sur $b_{k}$ par l'homomorphismed'Hurewicz $h: M U_{*} \rightarrow H_{*}(M U ;)$ Ialors :

$$
\mathcal{C}_{*}^{\varphi}\left(b_{k}\right)=\varphi\left(\left\{M^{2 k}\right\}\right)=\varphi_{0}\left(\left\{M^{2 k}\right\}\right) u^{k}
$$

Ceci nous permet de retrouver l'expression du caractère de Todd telle que'lle est donnée dans ([6],Theorem4.6)

$$
\operatorname{th}(\alpha)=\sum_{E} \mu\left(\left(\chi s_{E}\right)\right) \bar{B}_{E} u^{|E|}
$$

en posant $|E|=\sum_{i} i e_{i}$ et $\bar{B}_{E}=\left(\frac{1}{2}\right)^{e_{1}}\left(\bar{B}_{1}\right) e_{2} 0^{e_{3}}\left(\bar{B}_{2}\right)^{e_{4}} \ldots 0^{e_{2 i+1}}\left(\bar{B}_{i+1}\right)^{e_{2 i+2}} \ldots$

b) Caractère elliptique

Nous adopterons les notations de [4] pour désigner la cohomologie elliptique:

$$
\mathcal{E} l l^{*}(X)=M U^{*} \otimes_{M U} \mathbb{X}[1 / 2]\left[\delta, \epsilon, \gamma^{-1}\right]
$$

avec $\gamma=\delta^{2}-\epsilon$. (On pourrait aussi prendre $\gamma=\epsilon$ ou $\left.\Delta=\epsilon\left(\delta^{2}-\epsilon\right)^{2}\right)$. $\mathcal{E} l l^{*}(X)$ est une théorie cohomologique $I$-orientée définie par le genre elliptique $\varphi_{\text {ell }}: M U_{*}[1 / 2]\left[\delta, \epsilon, \gamma^{-1}\right]$ dont le logarithme est donné par

$$
g_{e l l}(X)=\int_{0}^{X} \frac{d t}{\sqrt{1-2 \delta t^{2}+\epsilon t^{4}}}=\sum_{n \geq 0} \frac{P_{n}(\delta / \sqrt{\epsilon}) \sqrt{\epsilon}^{n}}{2 n+1} X^{2 n+1}
$$


où $P_{n}$ est le n-ième polynôme de Legendre.

Notons que l'anneau de base $\mathbb{Z}[1 / 2]\left[\delta, \epsilon, \gamma^{-1}\right]$ est gradué, et que sa graduation est donnée par $d^{0} \delta=4$ et $d^{0} \epsilon=8$, il est donc $4 I$-gradué. De plus, on peut montrer (voir [3]) qu'enposant $\gamma=\delta^{2}-\epsilon$ et $\mathcal{A}_{*}=Q\left[\delta, \epsilon, \gamma^{-1}\right]$, alors la $I$ algèbre graduée $\mathcal{A}_{*}$ est complètement déterminée par $\delta$, $\gamma$ et $\mathcal{A}_{o}=\mathscr{Q}\left[\delta^{2} / \gamma\right]$. Pour tout knous avons: $\mathcal{A}_{8 k}=\gamma^{k} \mathcal{A}_{0}, \mathcal{A}_{8 k+1}=\gamma^{k} \delta \mathcal{A}_{o}=\gamma^{k} \mathcal{A}_{4 k}$. Par ailleurs, le caractère elliptique a été défini dans [3] par $e: M U_{*}(X) \rightarrow H_{*}\left(X ; A_{*}\right)$. La transformation $\mathcal{C}_{*}^{\varphi}$ dans le cas où $h^{*}(-)=\mathcal{E} l l^{*}(-)$ devient:

$$
e_{*}: H_{*}(M U ;) \rightarrow H_{*}\left(\mathcal{H} A_{*} ;\right) I A A_{*}=\Phi\left[\delta, \epsilon, \gamma^{-1}\right]
$$

et $\mathrm{d}^{\prime}$ aprés $(3): \mathrm{e}_{*}\left(b_{k}\right)=a_{k}^{\text {ell }}$ où $\sum_{k \leq 0} a_{k}^{\text {ell }} X^{k}$ coïncide avec la série caractéristique du genre elliptique donnée par (voir [8]):

$$
Q^{e l l}(X)=1-\sum_{k>0} \frac{G_{2 k}^{*}}{2^{2 k-2}(2 k-1) !}
$$

Il en découle que:

$$
\begin{aligned}
& a_{0}^{\text {ell }}=1 \\
& a_{2 k+1}^{\text {ell }}=0 \\
& a_{2 k}^{\text {ell }}=H_{k}=-\frac{G_{2 k}^{*}}{2^{2 k-2}(2 k-1) !}
\end{aligned}
$$

Si on pose $\tilde{H}_{E}=0^{e_{1}}\left(H_{1}\right)^{e_{2}} 0^{e_{3}}\left(H_{2}\right)^{e_{4}} \ldots 0^{e_{2 i+1}}\left(H_{i+1}\right)^{e_{2 i+2}} \ldots$ où $E=$ $\left(e_{1}, e_{2}, \ldots\right)$ on a :

$$
e_{*}\left(b_{E}\right)=\prod_{i \geq 1}\left(e_{*}\left(b_{i}\right)\right)^{e_{i}}=\tilde{H}_{E}
$$

on obtient alors

Proposition 4.2 : Pour tout $\alpha \in M U_{*}(X), e(\alpha)=\sum_{E} \mu\left(\left(\chi^{s} E\right)(\alpha)\right) \tilde{H}_{E}$

Démonstration : Immédiat à partir de (6) est du théorème 2.4 .

Remarque 4.3 Comme $e_{*}$ est un homomorphisme à valeurs dans $\mathcal{A}_{*}=$ $\mathscr{Q}\left[\delta, \epsilon, \gamma^{-1}\right]$ nous espérions obtenir une expression de $e_{*}\left(b_{i}\right)$ en fonction de $\delta$, $\epsilon$ et $\gamma$, ce qui est d'ailleurs immédiat dans le cas de la K-théorie, où $\mathcal{A}_{*}=\boldsymbol{Z}\left[u, u^{-1}\right]$ avec $d^{0} u=2$ (c.f remarque 4.1). Dans le cas du caractère 
elliptique, on peut noter que $e_{*}: H_{k}\left(M U ; I \rightarrow \mathcal{A}_{k}\right.$ est nul sauf si $k=0 \bmod -$ ulo 4; et en vertue de la proposition 3.1 on a $e_{*}(x)=\left\langle\Phi^{*} E_{k}\left(p_{1}, \ldots, p_{k}\right) ; x\right\rangle$ pour tout $x \in H_{*}(M U ;)$ Ioù les $E_{k}\left(p_{1}, \ldots, p_{k}\right)$ sont les polynômes elliptiques associés à la classe exponentielle elliptique (c.f [3]).

Si $k=2 n$ :

Nous considérons les polynômes :

$$
R_{2 n}\left(p_{1}, \ldots, p_{2 n}\right)\left[\delta^{2} / \gamma\right]=\frac{E_{2 n}\left(p_{1}, \ldots, p_{2 n}\right)}{\gamma^{n}}
$$

qui sont des polynômes de degré $2 \mathrm{n}$ en $\left(\delta^{2} / \gamma\right)$ à coefficients des polynômes homogènes en $p_{1}, p_{2} \ldots$ de poids $2 \mathrm{n}$. L'homomorphisme

$$
e_{*}: H_{8 n}\left(M U ; I \rightarrow \mathcal{A}_{8 n} \AA Q \Phi\left[\delta^{2} / \gamma\right] \gamma^{2}\right.
$$

sera donc défini par

$$
e_{*}(x)=\left\langle\Phi^{*} R_{2 n}\left(p_{1}, \ldots, p_{2 n}\right)\left[\delta^{2} / \gamma\right] ; x\right\rangle \gamma^{n}
$$

Si $\mathrm{k}=2 \mathrm{n}+1$ on introduit les polynômes

$$
S_{2 n+1}\left(p_{1}, \ldots, p_{2 n+1}\right)\left[\delta^{2} / \gamma\right]=\frac{E_{2 n+1}\left(p_{1}, \ldots, p_{2 n+1}\right)}{\delta \gamma^{n}}
$$

qui sont aussi des polynômes de degré $2 n+1$ en $\delta^{2} / \gamma$ à coefficients des polynômes homogènes en $p_{1}, p_{2}, \ldots$ de poids $2 n+1$.

$e_{*}: H_{8 n+4}\left(M U ; I \rightarrow \mathcal{A}_{8 n+4} \AA \mathscr{Q}\left[\delta^{2} / \gamma\right] \delta \gamma^{n}\right.$ est donné par

$$
e_{*}(x)=\left\langle\Phi^{*} S_{2 n+1}\left(p_{1}, \ldots, p_{2 n+1}\right)\left[\delta^{2} / \gamma\right] ; x\right\rangle \delta \gamma^{n} .
$$

En posant $r_{2 n}(x)=\left\langle\Phi^{*} R_{2 n}\left(p_{1}, \ldots, p_{2 n}\right)\left[\delta^{2} / \gamma\right] ; x\right\rangle \in \mathcal{A}_{o}=Q\left[\delta^{2} / \gamma\right]$ et $s_{2 n+1}(x)=$ $\left\langle\Phi^{*} S_{2 n+1}\left(p_{1}, \ldots, p_{2 n+1}\right)\left[\delta^{2} / \gamma\right] ; x\right\rangle \in \delta \mathcal{A}_{o}$, on obtient une autre formulation de (5) :

$$
e_{*}\left(b_{i}\right)=\left\{\begin{array}{lll}
0 & \text { si } & i \neq 0 \bmod (4) \\
r_{2 n}\left(b_{i}\right) \gamma^{n} & \text { si } & i=8 n \\
s_{2 n+1} & \text { si } & i=8 n+4
\end{array}\right.
$$




\section{Références :}

[1] V.M. Bukhshtaber, Characteristic classes in Cobordism and topological applicatiopns of the theory of single-valued and two-valued formal groups, English transl. in J. Soviet Math. 11, no.6, (1979).

[2] F. Hirzebruch, Topological Methods in Algebraic Geometry, 3rd edition Berlin-Heidelberg, Springer (1966).

[3] A. Kheldouni and F. Lamrini, Chern-Dold Character in Elliptic Cohomology,Extracta Mathematicae, vol.10 Nùm.2, 1-5, (1995).

[4] P.S. Landweber, Elliptic Curves and Modular Forms, L.N.M. 1326, Berlin-Heidelberg, Springer (1988).

[5] P.S. Landweber, Homological Properties of Comodules over $M U^{*}(M U)$ and $B P^{*}(B P)$, American J. of Math. vol. 98 no.3 pp 591-610.

[6] L. Smith, The Todd Character and Cohomology Operations, Advances in Mathematics, 10 (1973), pp 72-92.

[7] R.E. Stong, Notes on Cobordism Theory, McGraw-Hill, (1968).

[8] D. Zagier, Note on the Landweber-Stong elliptic genus, L.N.M. 1326, Berlin-Heidelberg, Springer (1988).

Received : October 1997.

\section{Abdelaziz Kheldouni \\ and}

Fayçal Lamrini

Université Mohammed Benabdallah

Faculté des Sciences Dhar-Mehraz

Département de Mathématiques

B. P. 1796 FES - Atlas

MAROC 